\title{
O paradoxo de bootstrap e o efeito ovo-galinha em um referencial superluminal
}

\author{
Colaboração Física Aberta ${ }^{1}$ \\ 27 de Novembro de 2021
}

\begin{abstract}
Resumo
Discutimos sobre o paradoxo de bootstrap e sobre o paradoxo ovo-galinha sob a perspectiva de um referencial mais rápido do que a luz.
\end{abstract}

palavras-chave: paradoxo de bootstrap, causalidade, velocidade superluminal, ovo-galinha

A versão mais atualizada deste artigo está disponivel em

https://osf.io/zuckf/download

https://zenodo.org/record/5699167

\section{Introdução}

1. Até o presente momento, não existe uma prova de que viagens no tempo sejam impossíveis.

2. Aliás, a teoria da relatividade especial garante ser possível viagens para o futuro $[1,2]$.

3. Resta, portanto, desvendarmos se é possível viajar ao passado [3].

${ }^{1}$ Todos os autores com suas afiliações aparecem no final deste artigo. 


\section{O Paradoxo de Bootstrap}

4. O paradoxo de bootstrap [4] pode ser ilustrado pelo seguinte exemplo.

5. M é um indivíduo que, no instante $t_{0}<t_{1}$ vai para o futuro $\left(t_{1}\right)$, pega uma fatia de pizza de si próprio (em $t_{1}$ ) e traz a pizza de volta para $t_{0}$.

6. Este exemplo deixa a entender que a pizza é a causa e o efeito de sua própria existência.

7. A pergunta que fica é: De onde surgiu a pizza?

8. Na próxima seção, iremos revisitar o exemplo (5) e descrever cada um dos eventos importantes.
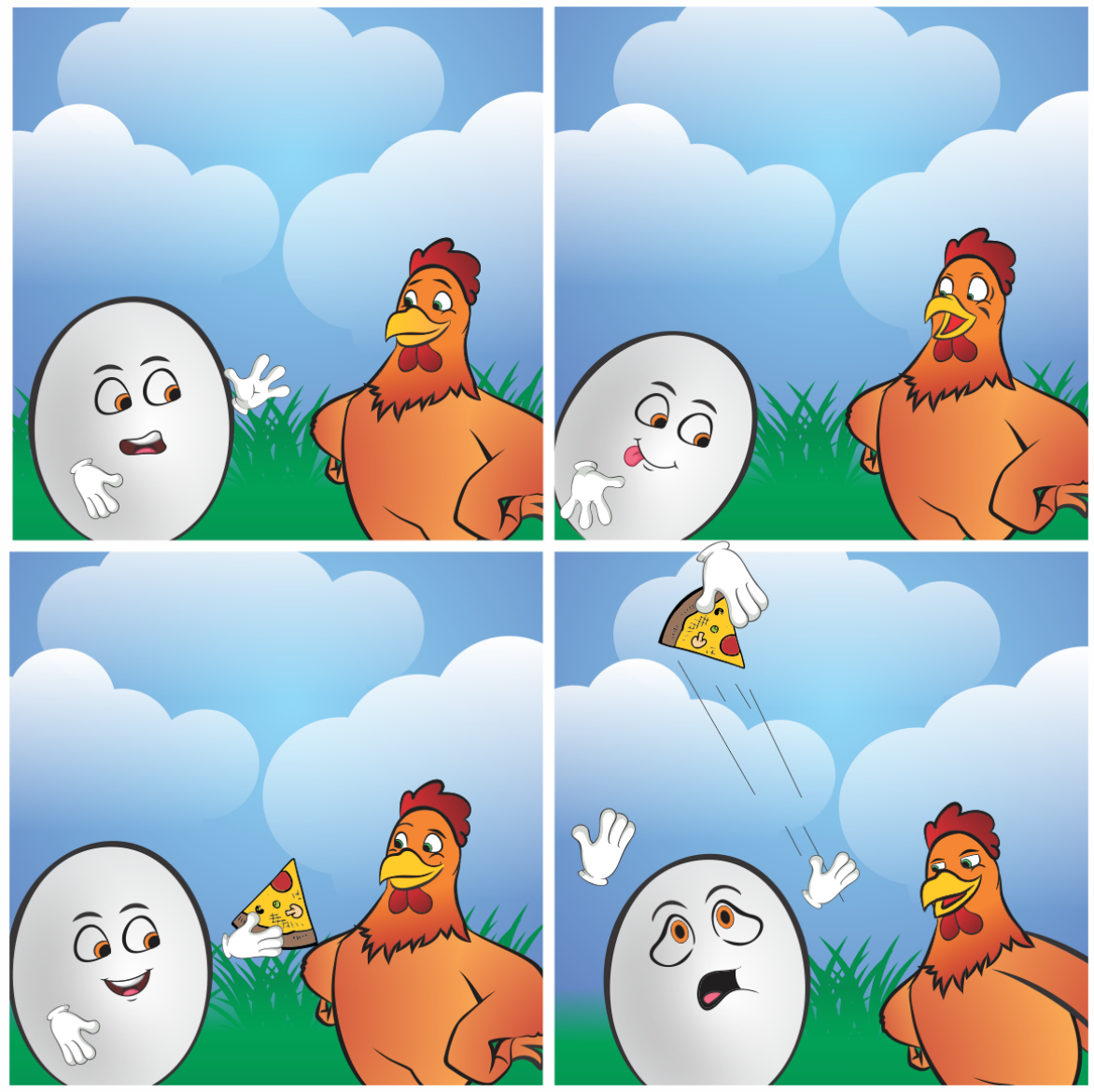

Figura 1: Ilustração do paradoxo de bootstrap. 


\section{Trazendo uma pizza do futuro}

9. O indivíduo $M$ mora em um planeta em que um pedido de pizza leva um ano para ser entregue, medido em seu próprio referencial.

10. Sejam os instantes $t_{0}<t_{1}<t_{2}<t_{3}<t_{4}<t_{5}$, medidos no referencial $M$.

11. Considere os seguintes eventos:

(A) em $t_{0}, M$ pede uma pizza (que chegará em $t_{4}$ );

$(B)$ em $t_{1}, M$ viaja para o futuro $\left(t_{5}\right)$, pega a pizza e retorna para o instante $t_{2}$

$(C)$ em $t_{3}, M$ come a pizza;

$(D)$ em $t_{4}$, a pizza chega;

$(E)$ em $t_{5}$, a pizza desaparece.

12. Note que, aqui, a causalidade está invertida, isto é, o efeito de comer a pizza (evento $C$ ) vem antes da causa (a chegada da pizza, evento $D)$.

13. Por $A<B$, entenda que o evento $A$ precede o evento $B$.

\section{Velocidade Superluminal}

14. Considere um referencial $S$ com velocidade constante $v>c$ com relação a $M$.

15. $c$ é a velocidade da luz.

16. A teoria da relatividade especial prevê que se algo pudesse viajar mais rápido do que a luz, então a ordem da causalidade seria invertida [1].

17. Em (11), $C<D$, isto é, a pizza é degustada (evento $C$ ) antes que ela chegue (evento $D$ ). 
18. É possível mostrar que, no referencial $S$, teremos $D<C$, isto é, primeiro a pizza chega e somente depois ela é degustada.

\section{Como detectar um bootstrap?}

19. Suponha que a Federação Intergaláctica de algum universo paralelo proíba paradoxos do tipo bootstrap.

20. Seria possivel detectar um viajante no tempo que causasse um bootstrap?

21. Vamos ver como responder (20).

22. Note que o bootstrap está presente apenas nos eventos $B, C$ e $E$ de (11).

23. Um sistema sem o bootstrap teria os seguintes eventos:

$(H)$ o sujeito pede a pizza;
$(I)$ a pizza chega;
$(J)$ a pessoa come a pizza.

24. Um observador em $S$ enxerga os eventos em ordem reversa, conforme mencionado em (16).

25. Assim, um observador em $S$ vê (23) na seguinte ordem: $J, I, H$.

26. Considerando o bootstrap do sistema (11), de acordo com o referencial $S$, a pizza primeiro aparece (evento $E$ ); em seguida, a pizza chega $(D) ; M$ come a pizza $(C)$; e, por fim, o pedido da pizza é feito $(A)$.

27. Olhando apenas para os eventos $D$ e $C$ de (11), $S$ percebe uma ordem usual de causa e efeito, isto é, primeiro a pizza chega (evento $D$ ), depois ela é degustada $(C)$. 
28. Como $S$ é um referencial superluminal, seria esperado ver uma ordem inversa de causalidade nos eventos sem bootstrap.

29. Assim, (27) representa uma evidência definitiva de que $M$ gerou um paradoxo do tipo bootstrap.

30. Portanto, com base no exposto neste experimento hipotético: Sim, seria possivel detectar um paradoxo do tipo bootstrap no referencial $S$ (com velocidade constante $v>c$ ).

\section{O paradoxo ovo-galinha}

31. Quem veio primeiro, o ovo ou a galinha?

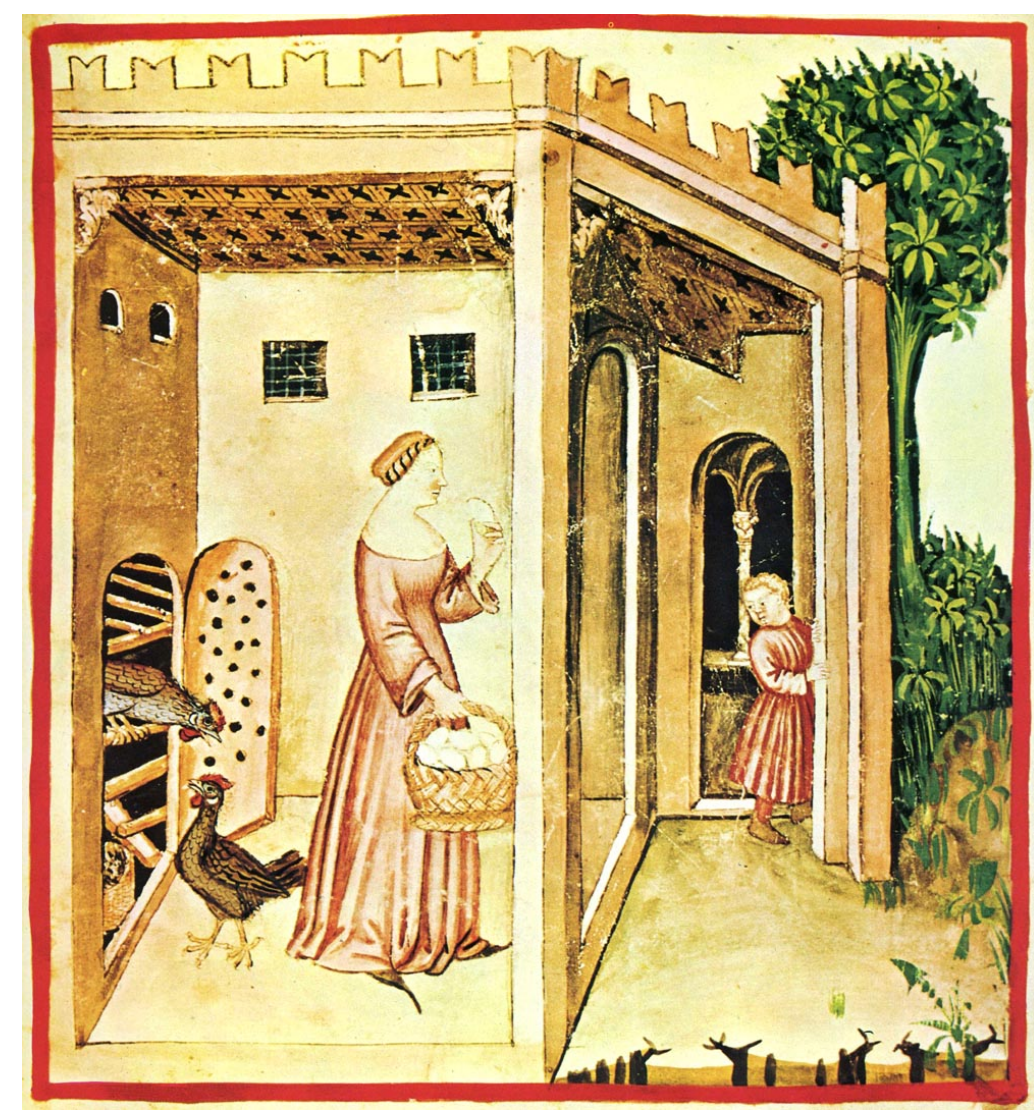

Figura 2: [5].

32. Considere os seguintes eventos:

$(G)$ a galinha nasce; 

$(O)$ a galinha bota um ovo.

33. No referencial da galinha (velocidade constante $v<c$ ), $G<O$.

34. No referencial $S$, (14), $O<G$.

\section{E agora?}

35. Apresentaremos as transformações de Lorentz e, em seguida, mostraremos a inversão da causalidade em um referencial superluminal.

\section{Transformações de Lorentz}

36. A derivação das transformações de Lorentz está disponível em [1].

37. Vamos trabalhar com o sistema natural de unidades em que a velocidade da luz $c=1[1,6]$.

38. Estamos considerando dois sistemas de referência, um laboratório na Terra, por exemplo, e um foguete com velocidade constante $v$ com relação ao laboratório.

39. O fator de contração de Lorentz é dado por

$$
\gamma=\frac{1}{\sqrt{1-v^{2}}} .
$$

40. Por simplicidade, vamos considerar eventos ocorrendo em apenas uma dimensão espacial.

41. $x$ é a coordenada espacial no referencial do laboratório $(L)$.

42. $x^{\prime}$ é a coordenada espacial no referencial do foguete $(F)$.

43. $t$ é a coordenada temporal no referencial do laboratório $(L)$.

44. $t^{\prime}$ é a coordenada temporal no referencial do foguete $(F)$. 
45. A equação que fornece o tempo de um evento no referencial do foguete, com relação à posição e ao tempo do evento no referencial do laboratório, é dada por

$$
t^{\prime}=-v \gamma x+\gamma t
$$

\section{Por que em um referencial superluminal, a causalidade é invertida?}

46. Seguindo a notação da seção anterior, seja $v=0,6$ a velocidade do foguete com relação ao labortatório e $v=3$ a velocidade do referencial superluminal $S$ com relação ao laboratório.

47. Por simplicidade, omitimos as unidades de tempo e espaço.

48. $x=2$ significa "duas unidades de espaço".

49. $t=3$ significa "três unidades de tempo".

50. Suponha os eventos 0, 1, 2 e 3, conforme a Fig. 3, com
(0) $x_{0}=0$,
(1) $x_{1}=1$,
(2) $x_{2}=2$,
(3) $x_{3}=3$.

51. Para $v=0,6$ (velocidade do foguete), temos $\gamma=1,25$.

52. Utilizando (45), temos que $t_{3}^{\prime}=-1$ e $t_{0}^{\prime}=0$ (Fig. 4).

53. Portanto, no referencial $F$, o evento 3 vem antes do evento 0.

54. Note que utilizamos três referenciais $(L, F, S)$. 


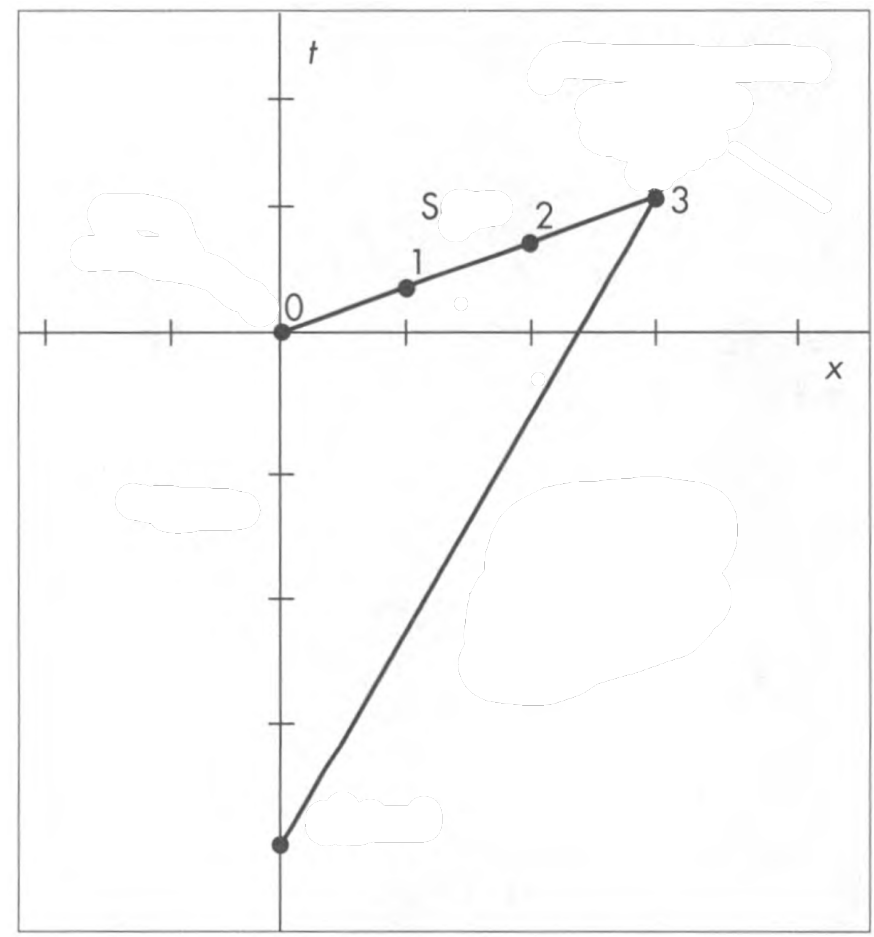

Figura 3: Diagrama do espaço-tempo no referencial do laboratório [1,2].

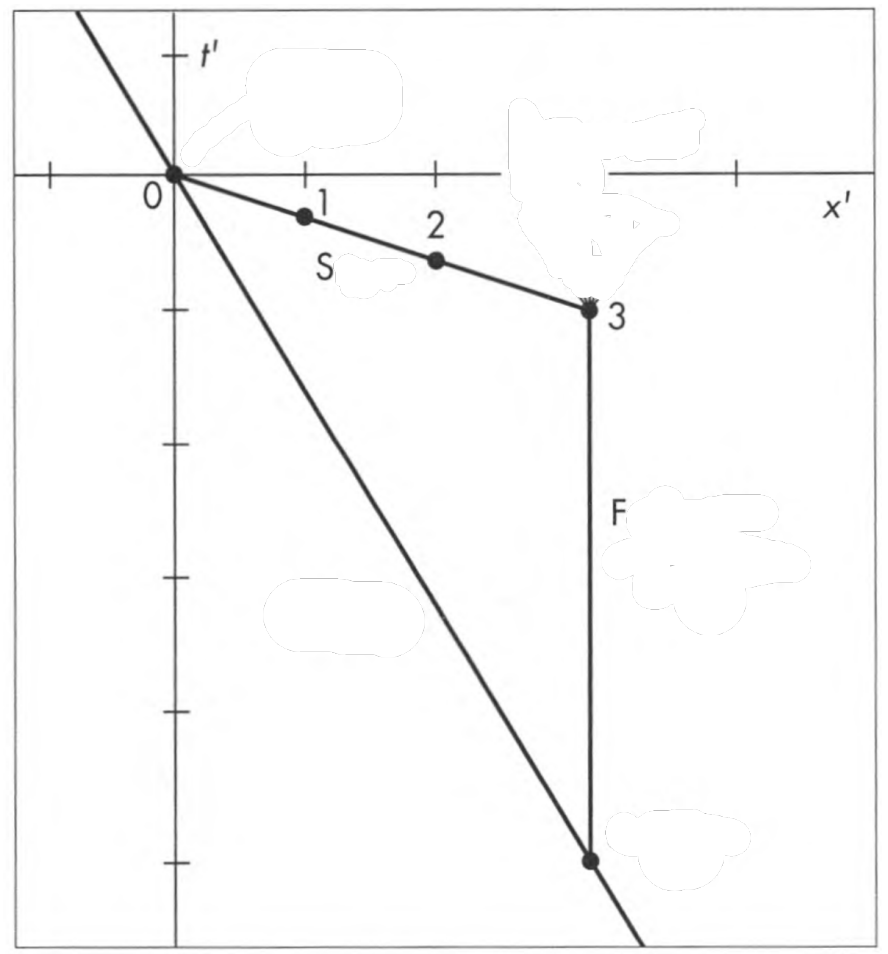

Figura 4: Diagrama do espaço-tempo no referencial do foguete $[1,2]$. 


\section{Tempo imaginário: um experimento mental}

55. Considere os seguintes eventos $\operatorname{com} x=0$ (na origem do laboratório):
(A) $t_{\mathcal{A}}=0$
(B) $t_{\mathcal{B}}=1$
(C) $t_{\mathcal{C}}=2$

56. Novamente, por simplicidade, omitimos as unidades de tempo e espaço.

57. O objetivo desta seção é analisarmos se há inversão de causalidade a partir de uma interpretação de tempo imaginário.

58. Seja a velocidade de um objeto voador não identificado (ovni) dada por $v=\sqrt{5}=2,236 \ldots$, isto é, pouco mais do que o dobro da velocidade da luz.

59. Assim, de (39),

$$
\gamma=\frac{1}{\sqrt{1-v^{2}}}=\frac{1}{\sqrt{1-5}}=\frac{1}{\sqrt{-4}}=-\frac{i}{2} .
$$

60. Lembrando que $i^{2}=-1$ e $i=\sqrt{-1}$.

61. A seguir, calculamos $t^{\prime}$ a partir de (45) para cada um dos eventos de (55):
(A) $t_{0}^{\prime}=-v \gamma x_{0}+\gamma t_{0}=0$
(B) $t_{1}^{\prime}=-v \gamma x_{1}+\gamma t_{1}=-\frac{i}{2}$,
(C) $t_{2}^{\prime}=-v \gamma x_{2}+\gamma t_{2}=-i$.

62. Um ansatz sobre como lidar com tempo imaginário é tomarmos seu módulo.

63. Tomando o módulo de (61), temos: 

(A) $\left|t_{0}^{\prime}\right|=0$,
(B) $\left|t_{1}^{\prime}\right|=\frac{1}{2}$,
$(\mathcal{C})\left|t_{2}^{\prime}\right|=1$

64. Portanto, neste cenário, considerando o módulo do tempo imaginário a partir das transformações de Lorentz, não houve inversão de causalidade neste experimento hipotético (mental).

\section{Considerações Finais}

65. Em uma versão modificada do paradoxo de bootstrap, tal como ilustramos em (11), o efeito vem antes da causa; já para um observador em um referencial superluminal, a causalidade é restabelecida.

66. Vale ressaltar que o limite da velocidade da luz é uma propriedade do espaço-tempo clássico.

67. Ainda não sabemos ao certo se o mundo quântico respeita (66).

68. Em [3], o leitor, cientista cidadão, poderá melhor compreender como paradoxos de viagens no tempo podem ser evitados, a partir da propriedade matemática da ortogonalidade; em outras palavras, se o tempo é ortogonal às três dimensões espaciais, então elas podem coexistir, considerando o contexto da teoria de muitos mundos (abordagem de Everett) [7].

\section{Ciência Aberta}

O arquivo latex para este artigo, juntamente com outros arquivos suplementares, estão disponíveis em [8]. Seja coautor(a) deste artigo, envie sua contribuição para mplobo@uft. edu.br. 


\section{Consentimento}

Os autores concordam com [9].

\section{Como citar este artigo?}

https://doi.org/10.31219/osf .io/zuckf

https://zenodo.org/record/5699167

\section{Licença}

CC-By Attribution 4.0 International [2]

\section{Referências}

[1] Taylor, Edwin F., Edwin F. Taylor, and John Archibald Wheeler. Spacetime Physics. Macmillan, 1992.

https://eftaylor.com/special.html

[2] CC. Creative Commons. CC-By Attribution 4.0 International. https://creativecommons .org/licenses/by/4.0

[3] Lobo, Matheus P. "Time Travel: Coexistence of Past, Present, and Future?." OSF Preprints, 2 Sept. 2019.

https://doi.org/10.31219/osf .io/7ruay

[4] Wikipedia. "Paradoxo de bootstrap".

https://pt.wikipedia.org/wiki/Paradoxo_de_bootstrap

[5] Wikipedia. "Tacuinum sanitatis."

https://pt.wikipedia.org/wiki/Tacuinum_sanitatis https://bit.1y/3c7twiX

[6] Lobo, Matheus P. "Measuring Time in Meters." OSF Preprints, 25 June 2019. https://doi .org/10.31219/osf .io/4ntwu 
[7] Carroll, Sean. Something deeply hidden: quantum worlds and the emergence of spacetime. Dutton, 2019.

[8] Lobo, Matheus P. "Open Journal of Mathematics and Physics (OJMP)." OSF, 21 Apr. 2020.

https://doi.org/10.17605/osf .io/6hzyp

[9] Lobo, Matheus P. "Simple Guidelines for Authors: Open Journal of Mathematics and Physics." OSF Preprints, 15 Nov. 2019.

https://doi.org/10.31219/osf .io/fk836

\section{Colaboração Física Aberta}

Matheus Pereira Lobo (autor principal, mplobo@uft.edu.br) $)^{1,2}$ https://orcid.org/0000-0003-4554-1372

Victor Manoel Soares da Conceição ${ }^{1}$

Matheus Santos Ribeiro ${ }^{3}$

Adriana da Silva Valadares ${ }^{1,4}$

Shirlei Nabarrete Deziderio ${ }^{1}$

${ }^{1}$ Universidade Federal do Tocantins (Brasil)

${ }^{2}$ Universidade Aberta (UAb, Portugal)

${ }^{3}$ Colaboração Aberta

${ }^{4}$ Escola Estadual Manoel Alves Grande (Campos Lindos, TO) 